\title{
Modelagem computacional de um trocador de calor solo-ar empregando diferentes modelagens para turbulência
}

Computational modeling of an earth-air heat exchanger using different modeling for turbulence

\author{
J. G. Ferraz ${ }^{1 *}$; B. R. Nunes ${ }^{1}$; M. K. Rodrigues ${ }^{1}$; R. S. Brum ${ }^{2}$; J. A. Souza ${ }^{1}$, \\ L. A. O. Rocha ${ }^{2}$; L. A. Isoldi ${ }^{1}$; E. D. dos Santos ${ }^{1}$ \\ ${ }^{1}$ Programa de Pós-Graduação em Modelagem Computacional (PPGMC), Escola de Engenharia (EE),Universidade \\ Federal do Rio Grande (FURG), 96.203-900, Rio Grande-RS, Brasil \\ ${ }^{2}$ Departamento de Engenharia Mecânica (DEMEC),Universidade Federal do Rio Grande do Sul (UFRGS), 90.050- \\ 170, Rio Grande-RS, Brasil \\ *juli-ferraz@hotmail.com \\ (Recebido em 15 de setembro de 2014; aceito em 29 de dezembro de 2014)
}

\begin{abstract}
A radiação solar é uma fonte de energia renovável e sua energia térmica pode ser aproveitada através da utilização de trocadores de calor solo-ar (TCSA) em ambientes construídos, podendo ser uma alternativa para reduzir o consumo de energia elétrica. Estes dispositivos consistem em dutos enterrados no solo, onde ocorre o escoamento do ar ambiente. $\mathrm{O}$ ar é resfriado/aquecido pelo solo nos períodos quente/frio podendo ser utilizado para climatizar ambientes construídos. Para simular numericamente os comportamentos térmico e fluidodinâmico de um TCSA é necessário empregar alguma modelagem para turbulência. Sendo assim, este trabalho tem como objetivo estudar diferentes modelos clássicos (URANS) para a abordagem numérica da turbulência: RSM (Reynolds Stress Model), $k-\varepsilon$ padrão, $k$ - $\omega$ padrão e Spalart Allmaras no estudo do princípio físico de funcionamento de um TCSA com um duto retilíneo, a fim de propor uma recomendação sobre os modelos mais adequados para esse tipo de aplicação. A modelagem computacional foi realizada através do software FLUENT que é baseado no método dos volumes finitos (MVF). Os resultados numéricos obtidos com os modelos URANS foram concordantes com os da literatura. Além disso, os modelos Spalart Allmaras e $k$ - $\omega$ padrão conduziram a uma redução de aproximadamente $40 \%$ no tempo de processamento em comparação ao RSM, sendo os mesmos recomendados para esse tipo de aplicação em TCSA com dutos retos.
\end{abstract}

Palavras-chave: modelagem computacional, modelos de turbulência e trocador de calor solo-ar.

Solar radiation is a source of renewable energy and its thermal energy can be exploited by using earth-air heat exchanger (EAHE) in built environments, could be an alternative to reduce electricity consumption. These devices consist of ducts buried in the soil, where the ambient air flow occurs. The air is cooled/heated by the soil in hot/cold periods can be used for cooling/heating of built environments. To numerically simulate the thermal and fluid dynamic behavior of an EAHE is necessary to use some modeling for turbulence. Thus, this work aims to study different turbulence modeling for approach of numerical approach of turbulence into URANS framework: RSM (Reynolds Stress Model), $k-\varepsilon$ standard, $k-\omega$ standard and Spalart Allmaras in the study of the main operational principle of an EAHE with a straight duct, in order to propose a recommendation about the most suitable models for this kind of application. The computational modeling was performed using the FLUENT software which is based on the finite volume method (FVM). The numerical results obtained with URANS models were in agreement with those depicted previously in literature. Furthermore, the Spalart Allmaras and $k-\omega$ standard models led to a reduction of almost $40 \%$ in time processing when compared with RSM, being that models recommended for this kind of application in EAHE.

Keywords: computational modeling, turbulence models and earth-air heat exchangers.

\section{INTRODUÇÃO}

Uma instalação de TCSA consiste em dutos enterrados no solo onde ocorre, com a utilização de ventiladores, o movimento forçado do ar exterior. Durante esse escoamento o ar troca calor com o solo, e sai do TCSA com uma temperatura mais baixa durante o verão e com uma temperatura mais elevada durante o inverno [1]. 
A utilização desse dispositivo pode ser considerada como uma alternativa promissora em custo e benefício para o ser humano, pois possibilita a construção de projetos de edificações com a utilização da energia solar que incide sobre a superfície do solo. Dessa forma, obtém-se um armazenamento de energia térmica nas camadas superficiais do solo. Em um ciclo anual, o solo acumula calor no período do verão e o libera durante o inverno [7], tornando a condição térmica em ambientes construídos mais agradável. Outra vantagem é a redução no consumo de energia elétrica relacionado ao uso de equipamentos de condicionamento de ar.

Normalmente, devido às características construtivas e operacionais, em um TCSA o escoamento do ar é turbulento. Dessa forma é necessário empregar alguma metodologia para a solução numérica destes escoamentos, visto que o tratamento dado ao escoamento laminar não é adequado para a solução de um escoamento turbulento [2]. As três principais metodologias para a solução numérica dos escoamentos turbulentos são a Simulação Numérica Direta (DNS - do inglês: Direct Numerical Simulation), a Simulação de Grandes Escalas (LES - do inglês: Large Eddy Simulation) e a modelagem clássica da turbulência em escoamentos transientes (URANS do inglês: Unsteady Reynolds-Averaged Navier Stokes) [2 - 5].

Neste trabalho, será utilizada a modelagem clássica da turbulência (URANS) que consiste na ideia de se realizar uma média temporal sobre as equações de conservação que modelam os escoamentos, sendo as variáveis do problema (velocidade, temperatura e pressão) decompostas em uma parcela média e outra flutuante no tempo [2]. Nesse processo são originadas novas incógnitas (tensões de Reynolds para a equação de conservação de quantidade de movimento e fluxos turbilhonares para a equação de conservação da energia) que contribuem para a predição do campo médio do escoamento. Contudo, o número de equações de conservação permanece o mesmo e passa-se a ter mais incógnitas do que equações, dando origem ao problema do fechamento $[2,5]$. Esses modelos são menos universais e precisam de ajustes para os diferentes escoamentos de base a serem simulados, possuindo dificuldades na abordagem de efeitos transientes como desprendimentos de vórtices, esteiras de vórtices e vórtices de KelvinHelmholtz em uma camada de mistura $[2,6]$. Contudo, essa metodologia tem sido satisfatória na predição de escoamentos internos. Outro fator que merece destaque é o seu amplo emprego na estimativa de parâmetros médios de escoamentos turbulentos e em problemas práticos de engenharia, visto que essa modelagem requer o menor esforço computacional entre as existentes.

Em se tratando de trocador de calor solo-ar existem diversas pesquisas envolvendo análises experimentais, analíticas e numéricas que podem ser facilmente encontradas na literatura, e.g. [8 -11]. Além dessas, são apresentadas referências que servem de embasamento teórico e fonte de dados experimentais e numéricos para a verificação e validação dos modelos de turbulência testados neste trabalho para o modelo reduzido empregado [1]. Contudo, não há uma recomendação sobre qual ou quais modelos são os mais recomendados para essa aplicação.

Em [7] e [12] foi realizado um estudo experimental e numérico sobre um TCSA, instalado no sul do Brasil, mais especificamente na cidade de Viamão, teve seu funcionamento monitorado e os resultados obtidos experimentalmente foram usados para validar o modelo computacional tridimensional desenvolvido. Nesse processo foi considerado o ciclo anual para as variações de temperaturas do solo e do ar na entrada e na saída do TCSA, mostrando diferenças inferiores a $15 \%$ entre os resultados numéricos e experimentais. O modelo computacional foi desenvolvido nos softwares GAMBIT e FLUENT. Nesses trabalhos o modelo de turbulência utilizado foi o modelo das tensões de Reynolds (Reynolds Stress Model - RSM), que é o modelo mais universal entre os modelos URANS.

No trabalho de [13] foi proposto um modelo numérico denominado Reduzido, que consistia em simular apenas uma parte do solo com o duto do TCSA inserido nesse domínio para obter os comportamentos térmico e fluido dinâmico de trocador de calor solo-ar. Com a utilização desse modelo foi evidenciada uma redução de até $45 \%$ no tempo de processamento em comparação com o modelo simplificado.

No âmbito da avaliação dos modelos de turbulência, em [14] foram comparados os modelos $k$ $-\varepsilon, k-\varepsilon$ realizable, $k-\omega$ SST e RSM para a predição de escoamentos internos em bombas de vácuo. Foi observado que o modelo $k-\omega$ SST conduziu aos resultados mais concordantes com predições experimentais. Em [15] os modelos $k-\varepsilon$ realizable, $k-\varepsilon$ para baixos números de 
Reynolds e RSM foram comparados para a predição do número de Nusselt em trocadores de calor com tubo helicoidal com vários passes. Nessa aplicação o modelo $k-\varepsilon$ para baixos números de Reynolds foi o recomendado para esse caso.

Conforme pode ser visto, não há um modelo de turbulência único recomendado para as diversas aplicações no campo da engenharia, bem como para a avaliação de trocadores de calor solo ar. Dentro deste contexto, o objetivo principal do presente trabalho é estudar os seguintes modelos de turbulência no âmbito da modelagem clássica (URANS): RSM, $k$ - $\varepsilon$ padrão, $k-\omega$ padrão e Spalart Allmaras, para a predição do comportamento térmico do princípio de funcionamento de um TCSA com um duto retilíneo, obtendo-se uma recomendação sobre os modelos mais indicados para o problema analisado. Com o intuito de reduzir o esforço computacional das simulações é empregado o modelo reduzido [1, 13].

\section{MODELAGEM MATEMÁTICA}

Para a avaliação do campo de temperatura transiente no solo é resolvida apenas a equação da energia [2] e [17] que é dada por:

$$
\frac{\partial T}{\partial t}=\frac{\partial}{\partial x_{j}}\left\{\alpha_{s} \frac{\partial T}{\partial x_{j}}\right\}
$$

onde $\alpha_{\mathrm{S}}$ é a difusividade térmica do solo $\left(\mathrm{m}^{2} / \mathrm{s}\right)$ e $T$ o campo de temperatura do solo (K).

Para a modelagem de escoamentos transientes, incompressíveis, com convecção forçada turbulenta no duto são resolvidas as equações de conservação de massa, quantidade de movimento e energia temporais médias (URANS). Essas equações são dadas por [2, 16]:

$$
\begin{array}{lr}
\frac{\partial \overline{v_{i}}}{\partial x_{i}}=0 & (i=1,2 \text { e } 3) \mathrm{em} t \times \Omega \\
\frac{\partial \overline{v_{i}}}{\partial t}+\frac{\partial\left(\overline{v_{i}} \bar{v}_{j}\right.}{\partial x_{j}}=-\frac{1}{\bar{\rho}} \frac{\partial \bar{p}}{\partial x_{j}} \delta_{i j}+\frac{\partial}{\partial x_{j}}\left\{v\left(\frac{\partial \overline{v_{i}}}{\partial x_{j}}+\frac{\partial \overline{v_{j}}}{\partial x_{i}}\right)-\tau_{i j}\right\} & (i, j=1,2 \mathrm{e} 3) \mathrm{em} t \times \Omega \\
\frac{\partial \bar{T}}{\partial t}+\frac{\partial}{\partial x_{j}}\left(\overline{v_{j} T}\right)=\frac{\partial}{\partial x_{j}}\left\{\alpha \frac{\partial \bar{T}}{\partial x_{j}}-q_{j}\right\} & (j=1,2 \text { e } 3) \mathrm{em} t \times \Omega
\end{array}
$$

onde ( ) representa parâmetros de média no tempo; $\rho$ é a massa específica do fluido $\left(\mathrm{kg} / \mathrm{m}^{3}\right) ; v$ é a viscosidade cinemática do ar $\left(\mathrm{m}^{2} / \mathrm{s}\right) ; \alpha$ é a difusividade térmica $\left(\mathrm{m}^{2} / \mathrm{s}\right) ; v_{i}$ é a velocidade na direção $i, i=1$, 2 e $3(\mathrm{~m} / \mathrm{s}) ; x_{i}$ é a coordenada espacial, $i=1,2$ e $3(\mathrm{~m}) ; p$ é a pressão $\left(\mathrm{N} / \mathrm{m}^{2}\right) ; T$ é a temperatura $(\mathrm{K}) ; \delta_{i j}$ é o delta de Kronecker; $\Omega$ é o domínio espacial $(\mathrm{m}) ; t$ representa o domínio temporal (s).

Os termos $\tau_{i j}$ e $q_{j}$ que surgem no processo de média temporal das equações de conservação da quantidade de movimento e energia, respectivamente, e precisam ser modeladas são dadas por:

$$
\begin{aligned}
& \tau_{i j}=\overline{v_{i}^{\prime} \overline{v_{j}^{\prime}}} \\
& q_{j}=\overline{v_{i}^{\prime}} \overline{T^{\prime}}
\end{aligned}
$$


sendo: $\tau_{i j}$ o tensor das tensões de Reynolds e $q_{j}$ o fluxo turbilhonar de energia.

\subsection{MODELAGEM CLÁSSICA DA TURBULÊNCIA (URANS)}

Para a solução do problema do fechamento e resolver os termos dados pelas Eqs. (5) e (6) são empregados os modelos de turbulência que consistem na solução de equações de transporte para as equações acima referidas, como é realizado no modelo RSM, ou em relacionar as Eqs. (5) e (6) com as taxas de deformação dos campos médios de velocidades e os gradientes de temperaturas médios no tempo empregando a hipótese de Boussinesq, o que é realizado nos demais modelos de turbulência empregados no presente trabalho [2, 5]. Por uma questão de simplicidade, neste estudo, serão apenas apresentadas as equações empregadas para resolver o problema de fechamento em cada modelo de turbulência. Maiores detalhes podem ser vistos em $[2,5,18]$. Aqui são resolvidos modelos mais simplificados com uma equação diferencial (Sparlat Allmaras) e modelos considerados mais completos a duas equações diferenciais $(k-\varepsilon \mathrm{e}$ $k-\omega)$.

\subsubsection{MODELO REYNOLDS STRESS MODEL (RSM)}

As equações de transporte das tensões de Reynolds, $\rho \overline{{\overline{u_{i}}}_{i} u_{j}^{\prime}}$, são definidas como [18]:

$$
\frac{\partial \tau_{i j}}{\partial t}+\bar{u}_{k} \frac{\partial \tau_{i j}}{\partial x_{k}}=-\left(\tau_{i k} \frac{\partial \bar{u}_{j}}{\partial x_{k}}+\tau_{j k} \frac{\partial \bar{u}_{i}}{\partial x_{k}}\right)-\frac{\partial}{\partial x_{k}} C_{i j k}+\Pi_{i j}-\varepsilon_{i j}+v \nabla^{2} \tau_{i j}
$$

onde: $C_{i j k}$ é o termo de difusão, $\Pi_{i j}$ a deformação da pressão e $\varepsilon_{i j}$ a dissipação, que são respectivamente, dados por:

$$
\begin{aligned}
& C_{i j k}=\overline{u_{i}^{\prime} u_{j}^{\prime} u_{k}^{\prime}}+\frac{1}{\rho}\left(\overline{p^{\prime} u_{i}^{\prime}} \delta_{j k}+\overline{p^{\prime} u_{j}^{\prime}} \delta_{i k}\right) \\
& \Pi_{i j}=\overline{\frac{p^{\prime}}{\rho}\left(\frac{\partial \bar{u}_{j}}{\partial x_{i}}+\frac{\partial \bar{u}_{i}}{\partial x_{j}}\right)} \\
& \varepsilon_{i j}=2 v \overline{\frac{\partial u_{i}}{\partial x_{k}} \frac{\partial u_{j}}{\partial x_{k}}}
\end{aligned}
$$

Os termos $C_{i j k} \Pi_{i j}$ e $\varepsilon_{i j}$ precisam ser modelados para completar o conjunto de equações governantes. Em [18] encontra-se uma abordagem mais detalhada a respeito dos termos da equação de transporte das tensões de Reynolds e suas hipóteses simplificativas.

\subsubsection{MODELO A UMA EQUAÇÃO DIFERENCIAL (SPALART ALLMARAS)}

A variável transportada no modelo Spalart Allmaras, $\tilde{v}$,é idêntica à viscosidade cinemática turbulenta exceto próximo a região da parede, onde uma função de parede é empregada para levar em consideração a anisotropia do escoamento nessa região. A equação de transporte para $\tilde{v}$ é dada por [17]: 


$$
\frac{\partial \tilde{v}}{\partial t}+\frac{\partial \tilde{v}}{\partial x_{i}} u_{i}=G_{v}+\frac{1}{\sigma_{\tilde{v}}}\left[\frac{\partial}{\partial x_{j}}\left\{(v+\tilde{v}) \frac{\partial \tilde{v}}{\partial x_{j}}\right\}+C_{b 2}\left(\frac{\partial \tilde{v}}{\partial x_{j}}\right)^{2}\right]-Y_{v}
$$

onde $G_{v}$ é a produção de viscosidade turbulenta e $Y_{v}$ é a destruição da viscosidade turbulenta que ocorre na região próxima da parede, devido ao bloqueio de parede e de amortecimento viscoso. $\sigma_{\widetilde{v}}=2 / 3$ e $C_{\mathrm{b} 2}=0,622$ são as constantes do modelo.

\subsubsection{MODELOS A DUAS EQUAÇÕES DIFERENCIAIS $(\boldsymbol{k}-\varepsilon \mathrm{E} \boldsymbol{k}-\boldsymbol{\omega})$}

Para este trabalho foi utilizado o modelo $k$ - $\varepsilon$ e $k$ - $\omega$ padrão, no qual a viscosidade turbulenta destes modelos é dada, respectivamente, por:

$$
\begin{aligned}
& v_{t}=C_{\mu} \frac{k^{2}}{\varepsilon} \\
& v_{t}=\alpha^{*} \frac{k}{\omega}
\end{aligned}
$$

sendo $C_{\mu}=0,09$ e $\alpha^{*}$ o coeficiente que amortece a viscosidade turbulenta fazendo uma correção de baixo número de Reynolds.

A equação da energia cinética da turbulência ( $k$ ) é representada por [19] e resolvida para os dois modelos de turbulência é dada por:

$$
\frac{\partial k}{\partial t}+\bar{v}_{j} \frac{\partial k}{\partial x_{j}}=\tau_{i j} \frac{\partial \bar{v}_{i}}{\partial x_{j}}+\frac{\partial}{\partial x_{j}}\left[\left(v+\frac{v_{t}}{\sigma_{k}}\right) \frac{\partial k}{\partial x_{j}}\right]-\varepsilon
$$

$\mathrm{E}$ as equações da dissipação de $\varepsilon$ e de $\omega$ (empregadas nos modelos $k-\varepsilon$ e $k-\omega$, respectivamente) são dadas por:

$$
\begin{aligned}
& \frac{\partial \varepsilon}{\partial t}+\bar{v}_{j} \frac{\partial \varepsilon}{\partial x_{j}}=\frac{\partial}{\partial x_{j}}\left[\left(v+\frac{v_{t}}{\sigma_{\varepsilon}}\right) \frac{\partial \varepsilon}{\partial x_{j}}\right]+C_{\varepsilon 1} \frac{\varepsilon}{k} \tau_{i j} \frac{\partial \bar{v}_{i}}{\partial x_{j}}-C_{\varepsilon 2} \frac{\varepsilon^{2}}{k} \\
& \frac{\partial \omega}{\partial t}+\bar{v}_{j} \frac{\partial \omega}{\partial x_{j}}=\frac{\partial}{\partial x_{j}}\left[\left(v+\frac{v_{t}}{\sigma_{\omega}}\right) \frac{\partial \omega}{\partial x_{j}}\right]+G_{\omega}-Y_{\omega}
\end{aligned}
$$

onde $C_{\varepsilon 1}=1,44, C_{\varepsilon 2}=1,92, \sigma_{\mathrm{k}}=1,0$ e $\sigma_{\varepsilon}=1,3$ para $\varepsilon$ e $\sigma_{\mathrm{k}}=2,0$ e $\sigma_{\omega}=2,0$ para $\omega$.

$\mathrm{O}$ primeiro termo que corresponde ao lado direito da Equação (14) é o de produção da energia cinética da turbulência, já o segundo termo é o transporte de $k$ por difusão e o último é a taxa de dissipação viscosa de $k$. Na Equação (15) os três termos do lado direito são, respectivamente, os mecanismos de difusão, produção e destruição de $\varepsilon$ [20]. Na Eq. (16) o termo $G_{\omega}$ e $Y_{\omega}$ representam, respectivamente, a geração e dissipação de $\omega$.

\section{MODELAGEM NUMÉRICA}

A simulação numérica para escoamentos de fluido, transferência de calor e problemas associados é conhecida como Dinâmica dos Fluidos Computacional, ou originalmente Computational Fluid Dynamics (CFD). Segundo [18] a CFD apresenta vantagens como: 
redução de tempo e custos para novos estudos, capacidade em estudar sistemas com análises complexas e o alto nível de detalhes nos resultados.

Para a simulação numérica desse trabalho foram utilizados os softwares GAMBIT para fase do pré-processamento (definição do domínio computacional, geração de malha) e o FLUENT para as etapas do processamento e pós-processamento. Esses softwares possibilitam resolver numericamente, em regime transiente, o modelo matemático para determinar a distribuição de temperatura do ar que flui nos TCSA e do solo em torno dos dutos.

Cabe destacar que o software FLUENT é baseado no MVF e, portanto, satisfaz os princípios de conservação em níveis discretos, ou seja, as equações da conservação de massa, quantidade de movimento e energia em nível de volumes finitos [21, 22].

Foi utilizado neste estudo o esquema de advecção upwind, nos termos advectivos, onde se leva em consideração a direção do escoamento para realizar o cálculo de uma determinada variável (velocidade, temperatura) na face do volume finito. Além disso, é garantida a positividade dos coeficientes dos termos advectivos, sendo assim, as soluções numéricas estão ausentes de oscilações numéricas [18].

O FLUENT contém cinco algoritmos de acoplamento pressão-velocidade: SIMPLE, SIMPLEC, PISO, Coupled e Fractional Step, todos utilizam o modelo de solução segregado, com exceção do Coupled que utiliza o acoplado. Neste trabalho foi utilizado o algoritmo Coupled, pois é um modelo robusto, apresenta um desempenho superior a algoritmos de solução segregados, e também é indicado para escoamentos transientes quando a qualidade da malha é baixa ou quando são apresentados grandes passos de tempo.

Para a equação da continuidade a solução foi considerada convergida para resíduo de $R=1,0$ $\times 10^{-4}$ enquanto para as equações de velocidades, energia e as equações adicionais em todos os modelos de turbulência foram considerados resíduos de $R=1,0 \times 10^{-6}$.

No processo de discretização temporal foi considerado um passo de tempo de 3600s (uma hora), com um número máximo de 200 iterações por passo de tempo, e um tempo final de dois anos. Como o primeiro ano é para estabilização da distribuição de temperatura no solo, então foram utilizados somente os resultados referentes ao segundo ano da simulação numérica para a realização das análises necessárias. Com relação à discretização espacial, foi gerada uma discretização espacial com 549.045 volumes tetraédricos, sendo 176.783 volumes no duto e 372.262 no solo.

Para simulação numérica desses casos foi utilizado um computador com processador Intel i7 Six Core e com memória de 24GB.

\section{DESCRIÇÃO DO PROBLEMA ABORDADO}

A geometria considerada para o TCSA trata-se de uma geometria simples de um duto reto, com comprimento $W=26,0 \mathrm{~m}$, altura $H=2,0 \mathrm{~m}$ de altura e largura $L=2,0 \mathrm{~m}$, como mostra a Figura 1. Foi utilizado neste trabalho o modelo computacional tridimensional Reduzido de [1] e [13] para representar um TCSA instalado a uma profundidade $p=1,6 \mathrm{~m}$. A superfície superior do solo foi denominada de $\mathrm{T}_{\mathrm{SUP}}$ e a inferior de $\mathrm{T}_{\mathrm{INF}}$, e para o diâmetro do duto foi usado $D=$ $110 \mathrm{~mm}$ e a espessura das paredes no duto não foi considerada nesse trabalho.

Com relação às condições do problema, o movimento do fluido é ocasionado pela imposição de um perfil de velocidades constante na entrada do duto de $v_{\text {in }}=3,3 \mathrm{~m} / \mathrm{s}$, enquanto que na saída do duto de ar é imposta uma pressão atmosférica $\left(p_{\text {out }}=1,0 \mathrm{~atm}\right)$. Nas superfícies do duto é imposta a condição de não deslizamento e impermeabilidade, i.e., $v_{1}=v_{2}=v_{3}=0,0 \mathrm{~m} / \mathrm{s}$. Com relação ao campo térmico, a variação térmica que ocorre no solo deve-se a incidência da radiação térmica sobre a superfície superior do solo. Em função disso, são impostas na superfície superior $\left(T_{\mathrm{SUP}}\right)$ e na superfície inferior do solo $\left(T_{\mathrm{INF}}\right)$ temperaturas dependentes do tempo para variar as condições do solo ao longo de um ano. As funções empregadas nas presentes simulações foram definidas anteriormente no trabalho de [1, 13]. Na saída do duto foi considerada uma condição de fluxo de calor nulo na direção do escoamento (localmente parabólico). 
As funções periódicas adotadas como condições de contorno para modelar as variações de temperatura na superfície superior e inferior do solo e do ar na entrada do duto, são definidas, respectivamente, como:

$$
\begin{aligned}
& T_{v}(\mathrm{t})=20,49+5,66 \operatorname{sen}\left(\left(1,78 \times 10^{-2} \mathrm{t}\right)-5,30\right) \\
& T_{S U P}(\mathrm{t})=18,70-4,8887 \operatorname{sen}\left(\left(1,73 \times 10^{-2} \mathrm{t}\right)-2,313\right) \\
& T_{I N F}(\mathrm{t})=18,70+2,25 \operatorname{sen}\left(\left(1,74 \times 10^{-2} \mathrm{t}\right)+0,01557\right)
\end{aligned}
$$

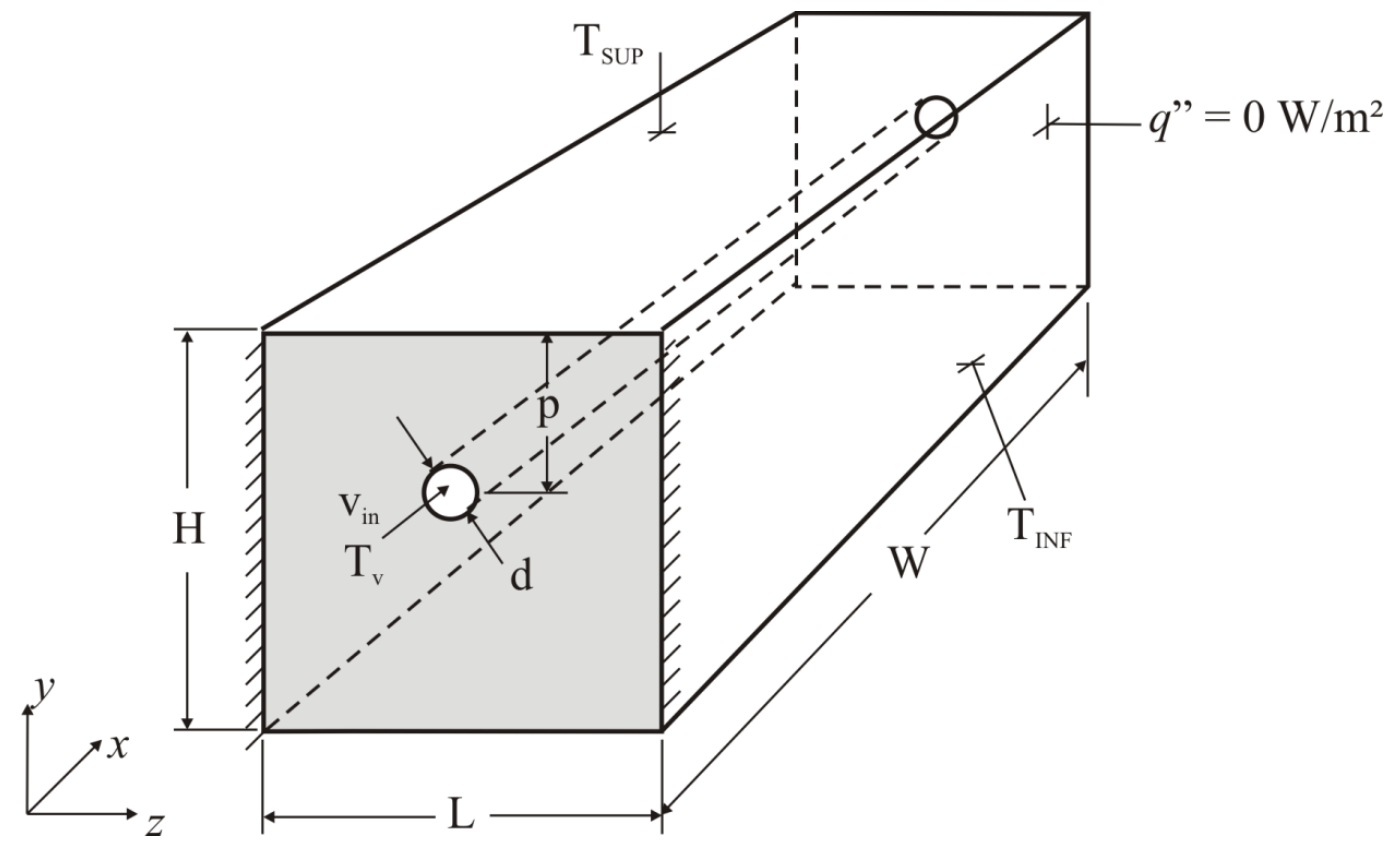

Figura 1: Domínio computacional do modelo reduzido com duto retilíneo.

Essas funções, que descrevem o ciclo anual de variação de temperatura, foram inseridas no FLUENT como Funções Definidas pelo Usuário, originalmente User Defined Functions (UDF). Além disso, cabe destacar que as Equações (18) e (19) foram obtidas através de um ajuste estatístico dos dados experimentais apresentado em Vaz [7].

Com relação à condição inicial do problema, foi usada à temperatura média anual do solo que corresponde a $291,7 \mathrm{~K}$, aproximadamente $18,7^{\circ} \mathrm{C}$. Além disso, as propriedades termofísicas do solo e do ar: $\rho$ (massa específica), $k$ (condutividade térmica) e $c_{p}$ (calor específico), conforme Tabela 1.

Tabela 1: Propriedade dos materiais.

\begin{tabular}{cccc}
\hline & \multicolumn{3}{c}{ Propriedade dos materiais. } \\
\cline { 2 - 4 } Componentes & $\rho\left(\mathbf{k g} / \mathbf{m}^{3}\right)$ & $k(\mathbf{W} / \mathbf{m K})$ & $C_{p}(\mathbf{J} / \mathbf{k g} / \mathbf{K})$ \\
\hline Solo Argiloso & 1800 & 2,1 & 1780 \\
Ar & 1,16 & 0,0242 & 1010 \\
\hline
\end{tabular}

\section{RESULTADOS E DISCUSSÃO}

A Figura 2 ilustra a comparação dos campos de temperatura na saída de ar do duto do TCSA obtidas para os modelos estudados no presente trabalho (RSM, $k-\varepsilon, k-\omega$ e Spalart Allmaras), os resultados experimentais e numéricos de [7] e os preditos com os modelos simplificado e reduzido de [1]. 
Observou-se que entre os modelos URANS os resultados foram extremamente concordantes. Além disso, também houve uma boa concordância com os resultados numéricos obtidos em $[1,13,23]$ utilizando o modelo RSM para os modelos simplificado e reduzido. É importante salientar que a geometria proposta neste estudo apresenta dimensões diferentes da apresentada em [13], i.e., o domínio empregado no presente estudo possui uma largura $L$ maior do que o estudado em [13], onde o valor de $L$ empregado foi $L=0,55 \mathrm{~m}$. Essa diferença acarreta em pequenas diferenças entre os resultados encontrados aqui e os preditos em [13].

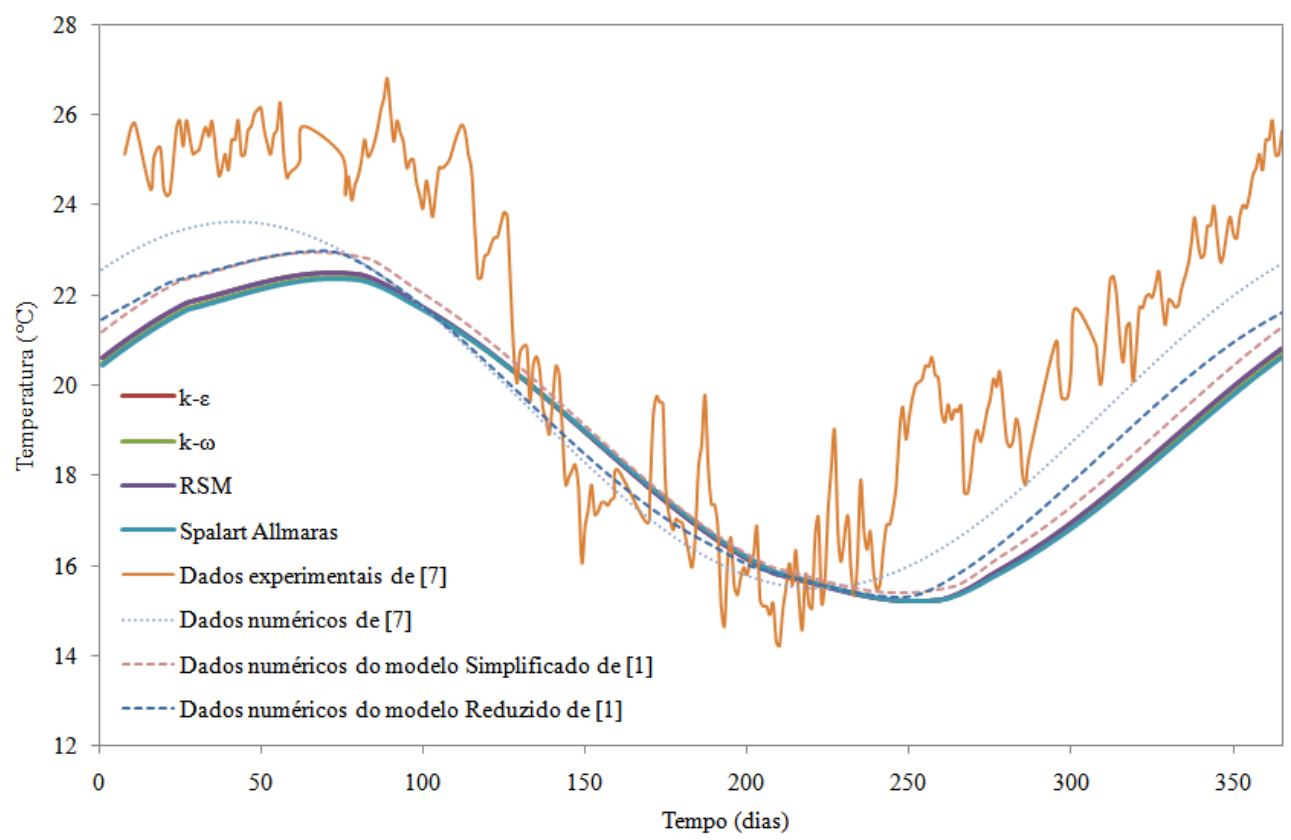

Figura 2: Comparação dos modelos de turbulência com dados experimentais e numéricos de [7] e com dados numéricos dos modelos: Simplificado e Reduzido de [1].

Comparando as soluções numéricas do presente trabalho com os dados experimentais e numéricos de [7], verifica-se que há uma boa concordância entre os mesmos, apresentando diferenças máximas de $4,0^{\circ} \mathrm{C}$ e $2,0^{\circ} \mathrm{C}$ em relação aos dados experimentais e numéricos.

Entre os resultados numéricos estudados os melhores resultados foram alcançados quando o domínio completo que reproduzia exatamente o domínio experimental e com 3 dutos enterrados foi simulado, i.e., os resultados obtidos em [7]. Posteriormente, verificou-se que o modelo reduzido com $L=2,0 \mathrm{~m}$ conduziu a piores resultados do que o modelo simplificado, o que era esperado. Além disso, foi observado que o uso de uma largura diferente para o modelo reduzido apresentou influência sobre o campo de temperaturas do ar na saída ao longo do ano, indicando a necessidade de futuros estudos sobre a influência desse parâmetro sobre a solução. Apesar dessas diferenças, de uma forma geral, os resultados numéricos foram satisfatoriamente concordantes com os resultados experimentais e numéricos obtidos com modelos completo e simplificado. Vale destacar que o uso do modelo reduzido conduziu a uma redução do esforço computacional e poderá ser empregado na recomendação teórica em geometrias complexas de TCSA.

Em relação ao tempo de simulação dos modelos de turbulência, pode-se observar que os modelos Spalart Allmaras e $k$ - $\omega$ apresentaram uma melhor eficiência, levando em consideração que estes modelos comparados ao RSM obtêm um ganho de $40 \%$ em tempo de processamento necessário para resolução do problema, como pode ser visto na Tabela 2. 
Tabela 2: Tempo de simulação dos modelos de turbulência.

\begin{tabular}{|c|c|c|}
\hline \multirow[b]{2}{*}{ Modelo } & \multicolumn{2}{|c|}{ Tempo de simulação dos modelos de turbulência } \\
\hline & Tempo de processamento (s) & Diferença (\%) \\
\hline Spalart Allmaras & 18000 & ---------- \\
\hline $\mathbf{k}-\omega$ & 18000 & ---- \\
\hline $\mathbf{k}-\varepsilon$ & 19800 & 10,0 \\
\hline RSM & 25200 & 40,0 \\
\hline
\end{tabular}

Na Tabela 3 foi comparada a temperatura de saída do duto dos modelos de turbulência com o dado experimental, o que mostra resultados semelhantes entre os modelos URANS. Os resultados obtidos com os modelos RSM e $k-\varepsilon$ foram apenas $0,5 \%$ e $0,8 \%$ melhores do que os obtidos com os modelos $k$ - $\omega$ e Spalart Allmaras, respectivamente. Dessa forma, os resultados indicam que para esse caso específico os melhores modelos acabam sendo definidos pelo tempo de processamento.

Tabela 3: Temperatura de saída dos modelos de turbulência e da solução experimental.

\begin{tabular}{|c|c|c|}
\hline \multirow[b]{2}{*}{ Solução } & \multicolumn{2}{|c|}{$\begin{array}{c}\text { Temperatura de saída dos modelos de turbulência e } \\
\text { da solução experimental }\end{array}$} \\
\hline & $\mathrm{T}_{\text {sai }}$ - saída do duto $\left({ }^{\circ} \mathrm{C}\right)$ & Diferença - $\mathbf{T}_{\text {med }}(\%)$ \\
\hline Experimental & 25,64 & \\
\hline Spalart Allmaras & 20,65 & 19,5 \\
\hline$k-\omega$ & 20,72 & 19,2 \\
\hline$k-\varepsilon$ & 20,84 & 18,7 \\
\hline RSM & 20,85 & 18,7 \\
\hline
\end{tabular}

\section{CONCLUSÃO}

Neste trabalho foi realizado um estudo numérico comparando diferentes modelos de turbulência empregados num TCSA com geometria simples: um duto reto, utilizando para modelagem computacional o modelo Reduzido de [1].

Os resultados mostraram que todos os modelos de turbulência apresentaram resultados muito semelhantes, além de concordantes com a literatura. E em relação ao tempo de processamento os modelos Spalart Allmaras e $k$ - $\omega$ obtiveram resultados igualmente satisfatórios. Contudo, o tempo de processamento requerido a esses modelos foi aproximadamente $40 \%$ inferior ao requerido com o modelo RSM, i.e., uma diferença proporcional à obtida quando comparados os modelos simplificado e reduzido.

Os resultados evidenciaram que o uso dos modelos Spalart Allmaras e $k$ - $\omega$ padrão são os mais indicados para a simulação de TCSA com dutos retos. Como proposta de continuidade deste estudo é sugerida a investigação de diferentes modelos de turbulência empregados em um TCSA com geometria complexa, utilizando o modelo de [1], a fim de obter novas recomendações para esse tipo de aplicação. Além disso, pretende-se estudar a viabilidade de empregar LES para a solução desse problema.

\section{AGRADECIMENTOS}

Os autores J. A. Souza, L. A. O. Rocha, L. A. Isoldi e E. D. dos Santos agradecem ao CNPq pelo auxílio financeiro. Os autores J. G. Ferraz e B. R. Nunes agradecem a CAPES pela bolsa de mestrado. 


\section{REFERÊNCIA BIBLIOGRÁFICAS}

1. Brum R. Modelagem Computacional De Trocadores De Calor Solo-Ar. Dissertação De Mestrado, Universidade Federal Do Rio Grande (Furg), Programa De Pós-Graduação Em Modelagem Computacional, Rio Grande, RS, Brasil, 2013. 137p.

2. Wilcox DC. Turbulence Modeling For CFD. Dcw Industries, USA, 2002. 540p.

3. Kasagi N, Iida O. Progress In Direct Numerical Simulation Of Turbulent Heat Transfer, Proceedings Of The 5th Asme/Jsme Joint Thermal Engineering Conference; 1999 Mar 15-19; San Diego, Usa.

4. Lesieur M, Métais O, Comte P. Large-Eddy Simulations Of Turbulence, Cambridge, New York, 2005. 232p.

5. Pope SB. Turbulent Flows, Cambridge University Press, New York, Usa, 2008. 802p.

6. Dos Santos ED. Análise Numérica De Escoamentos Turbulentos Não Reativos Com Transferência De Calor Por Convecção E Radiação Térmica Em Meios Participantes. Tese De Doutorado, Universidade Federal Do Rio Grande Do Sul (UFRGS), Programa De Pós-Graduação Em Engenharia Mecânica, Porto Alegre, RS, Brasil, 2011. 208p.

7. Vaz J. Estudo Experimental E Numérico Sobre O Uso Do Solo Como Reservatório De Energia Para O Aquecimento E Resfriamento De Ambientes Edificados. Phd Thesis, Universidade Federal Do Rio Grande Do Sul (UFRGS), Porto Alegre, RS, Brasil, 2011. 237p.

8. Mihalakakou G, Santamouris M, Asimakopoulos DN, Papanikolaou N. Impacto Of Ground Cover On The Efficiencies Of Earth-To-Air Heat Exchangers. Appl Energ. 1994; 48(1):19-32.

9. Hollmuller P, Lachal B. Cooling And Preheating With Buried Pipe Systems: Monitoring, Simulation And Economic Aspects. Energ Buildings. 2001; 33(5):509-518, doi:10.1016/S03787788(00)00105-5.

10. Wu H, Wang S, Zhu D. Modelling And Evaluation Of Cooling Capacity Of Earth-Air-Pipe Systems. Energ Convers Manage. 2007; 48(5): 1462-71, doi: 10.1016/j.enconman.2006.12.021.

11. Misra R, Bansal V, Agrawal GD, Mathur J, Aseri TK. Cfd Analysis Based Parametric Study Of Derating Factor For Earth Air Tunnel Heat Exchanger. Appl Energ. 2013; Mar;103: 266-277, doi: 10.1016/j.apenergy.2012.09.041.

12. Vaz J, Sattler MA, Dos Santos, ED, Isoldi LA. Experimental And Numerical Analysis Of An EarthAir Heat Exchanger. Energ Buildings. 2011; Sep;43(9):2476-82, doi: 10.1016/j.enbuild.2011.06.003.

13. Brum R, Rocha LA, Vaz J, Dos Santos ED, Isoldi LA. A New Computational Modeling To Predict The Behavior Of Earth-Air Heat Exchangers. Energ Buildings. 2013; Sep;64:395-402,doi: 10.1016/j.enbuild.2013.05.032.

14. Dong J, Wang X, Tu J. Numerical Research About The Internal Flow Of Steam-Jet Vacuum Pump: Evaluation Of Turbulence Models And Determination Of The Shock-Mixing Layer. Physics Procedia. 2012; 32: 614-322, doi: 10.1016/j.phpro.2012.03.608.

15. Lin WC, Ferng YM, Chieng CC. Numerical Computations On Flow And Heat Transfer Characteristics Of A Helically Coiled Heat Exchanger Using Different Turbulence Models. Nucl Eng Des. 2013 Oct; 263:77-86, doi: 10.1016/j.nucengdes.2013.03.051.

16. Bejan A. Convection Heat Transfer, John Willey, Durham, Usa, 2004. 685p.

17. Fluent 6.3, Documentation Manual. 2501p.

18. Versteeg HK, Malalasekera W. An Introduction To Computational Fluid Dynamics: The Finite Volume Method. England. 2 ed. Pearson Education, 2007. 520p.

19. Launder BE, Spalding DB. Lectures In Mathematical Models Of Turbulence. London: Academic Press; 1972. 169p.

20. Deschamps CJ. Turbulência. Volume I, In: Silva Freire AP, Menut PPM, Su J, editors. Associação Brasileira De Ciências Mecânicas, Rio de Janeiro, Brasil; 2002. 272p.

21. Maliska CR. Transferência De Calor E Mecânica Dos Fluidos Computacionais, Ltc - Livros Técnicos E Científicos Editora S.A. 2 ed. Rio De Janeiro; 2010. 472p.

22. Patankar SV. Numerical Heat Transfer And Fluid Flow. Mc Graw-Hill. New York. Usa;1980. 200p.

23. Brum R, Rocha LA, Vaz J, Dos Santos ED, Isoldi LA. Development Of Simplified Numerical Model For Evaluation Of The Influence Of Soil-Air Heat Exchanger Installation Depth Over Its Thermal Potential. Int. J Advanc Renew Energ Research 2012; 1 (9): 505-514. 